\title{
Description of Insulin Resistance and Beta-Cell Pancreas Dysfunction in Prediabetic Patients
}

\author{
Eva Decroli ${ }^{1}$, Yanne Pradwi Efendi ${ }^{2}$, Alexander Kam ${ }^{3}$, Asman Manaf ${ }^{4}$, Syafril Syahbuddin ${ }^{5}$ \\ \{dr.evadecroli@gmail.com ${ }^{1}$, yanne.efendi89@gmail.com², alexander_kam@yahoo.com ${ }^{3}$, \\ pibipd@yahoo.com ${ }^{4}$,ssyahbuddin@yahoo.com ${ }^{5}$ \} \\ Department of Internal Medicine, Faculty of Medicine, Andalas University - M. Djamil General \\ Hospital, Padang, Sumatera Barat
}

\begin{abstract}
Prediabetes is the forerunner of diabetes mellitus. Hence it has to be carefully cared for. Main conditions causing this are insulin resistance and beta cell dysfunction. Insulin resistance can be assessed by using several methods, one of which is performed by assessing the value of HOMA-IR. Beta cell function can be determined by assessing HOMA-B. In this research we aim to obtain the description of insulin resistance and beta cell pancreas dysfunction on prediabetic patients, using the analytic observational method with cross-sectional approach. HOMA-IR and HOMA-B were assessed in 20 prediabetic patients, and their values were obtained by examining basal insulin and fasting glucose level. We found man to woman ratio 1:1, with mean (SD) age 35.8 (5.7) years old, BMI $24.9(4.3) \mathrm{kg} / \mathrm{m} 2$, fasting blood glucose 104.8 (4.3) $\mathrm{mg} / \mathrm{dL}$, and blood glucose two hours after oral glucose tolerance test 121.7 (23.1) $\mathrm{mg} / \mathrm{dl}$. Mean of HOMA-IR on prediabetic patients was 4.1 (3.2) and mean of HOMA-B was 188.3 (155.1). The increase of insulin resistance on this study is by studies that were conducted by Nguyen and Mohtarin. The increase of HOMA-B in this study is following other studies by Mohtarin and Owei, supposedly due to beta cell compensation process against the increase of insulin resistance. We conclude that the increase of HOMA-IR in prediabetics is compensated for by an increase in HOMA-B.
\end{abstract}

Keywords: Prediabetes, HOMA IR, HOMA B.

\section{Introduction}

Prediabetic is a part of glycometabolic abnormality caused by insulin resistance and/or beta cell pancreas dysfunction [1]. During the progression of prediabetes, in early phase increasing level of blood glucose will be compensated for by the increasing the level of insulin so that glucose uptake will increase. Then, if there are increasing insulin resistance and/or pancreatic beta cell dysfunction, glucose uptake will decrease resulting in blood glucose level increase. This was described by DeFronzo in 2013 using a picture showing mean plasma glucose and mean plasma insulin levels during oral glucose tolerance test (OGTT) and insulin-mediated glucose uptake in five groups of people, namely lean-normoglycemic, obesity-normoglycemic, obesity-impaired glucose tolerance, obesity-diabetes high insulin, and obesity-diabetes low insulin people [2].

Maftin et al. in 2010 stated that failure of pancreatic beta cells had occurred at the earlier step. Another metabolic research concluded that in impaired glucose tolerance group, there have been maximal insulin resistance and loss of beta cell function in more than $80 \%$ [3]. 
Prediabetes is diagnosed from a laboratory parameter when serum blood glucose is higher than normal value, but not high enough for diabetes [4]. Prediabetes consists of impaired glucose tolerance (IGT) and impaired fasting glucose (IFG) level. IGT is defined as a condition when serum glucose level two hours after an oral glucose tolerance test is more than $140 \mathrm{mg} / \mathrm{dL}(7.8$ $\mathrm{mmol} / \mathrm{L})$ but less than $200 \mathrm{mg} / \mathrm{dL}(11.1 \mathrm{mmol} / \mathrm{L})$, and IFG is defined as a condition when fasting blood glucose level is $100 \mathrm{mg} / \mathrm{dL}(5.6 \mathrm{mmol} / \mathrm{L})$ or more but less than $126 \mathrm{mg} / \mathrm{dL}(7.0 \mathrm{mmol} / \mathrm{L})$ $[5,6]$.

Centers for Disease Control and Prevention in 2014 presumed that approximately 15-30\% prediabetic patient would become diabetes in five years. Nowadays, diabetes mellitus is a global burden and main health problem in the $21^{\text {st }}$ century. The prevalence has increased in the last decade. According to International Diabetes Federation Western Pacific Region (WPR) in 2015, of 415 million population of diabetes in the world, 153 million was found in Western Pacific and 10 million in Indonesia [1]. Prediabetic is the forerunner of diabetes mellitus. Hence it has to be carefully cared for $[5,6]$.

As the main process involved in the pathophysiology of prediabetes, insulin resistance, and beta-cell dysfunction need to be assessed, insulin resistance can be assessed using several methods, one of which is the value of HOMA-IR. Cobb et al. in 2013 concluded that HOMAIR was an important indicator of the development of prediabetes and diabetes mellitus [7]. Betacell dysfunction is shown by the failure of phase 1 insulin secretion in response to blood glucose. Beta cell dysfunction can be determined by assessing the value of HOMA-B. There are many studies about the role of HOMA-B in predicting diabetes $[8,9,10]$. This research aims to obtain the description of insulin resistance and beta cell pancreas dysfunction on prediabetic patients.

\section{Material and methods}

This research is an analytic observational study with the cross-sectional approach, in which an independent and dependent variable was assessed at the same time. Research was performed on the campus of Medical Faculty, Andalas University, Padang, Indonesia for six months. Subjects in this research were selected using random sampling method. Inclusion criteria were prediabetes with a family history of having the risk of type 2 diabetes mellitus, while exclusion criteria consisted of the patient with diabetes mellitus or prediabetes on medication.

Prediabetics that conform with inclusion and exclusion criteria was 20 patients. Data was collected regarding age, sex, body mass index (BMI), fasting blood glucose, and blood glucose level two hours after an oral glucose tolerance test. The assessment of HOMA-IR and HOMA$\mathrm{B}$ followed these. HOMA-IR was equal to fasting insulin in $\mathrm{mU} / \mathrm{L}$ times fasting blood glucose in $\mathrm{mmol} / \mathrm{L}$ divided by 22.5 , and HOMA- $\beta$ was equal to 20 times fasting insulin in $\mathrm{mU} / \mathrm{L}$ divided by (fasting blood glucose in $\mathrm{mmol} / \mathrm{L}$ subtracted by 3.5 )

\section{Results}

Means and standard deviations of subjects were obtained as follows: age was $35,75(5,68)$ years, the ratio of man to woman was $1: 1$, mean of BMI was $24,85(4,25) \mathrm{kg} / \mathrm{m}^{2}$, fasting blood glucose was 104,80 $(4,25) \mathrm{mg} / \mathrm{dL}$, and blood glucose level two hours after oral glucose tolerance test was $121,70(23,10) \mathrm{mg} / \mathrm{dL}$. Based on the age group, most of the subject $(50 \%)$ were in 31 40 years range, and most of the subjects $(50 \%)$ were in normal BMI group (Table 1$)$. 
Table 1. Characteristics of Prediabetic Patient

\begin{tabular}{lll}
\hline Characteristic & $\mathrm{n}(\%)$ & Mean (SD) \\
\hline Sex & $10(50 \%)$ & \\
Man & $10(50 \%)$ & \\
Woman & 20 & $35.75(5.68)$ \\
Age (year) & $4(20 \%)$ & \\
$21-30$ & $10(50 \%)$ & \\
$31-40$ & $6(30 \%)$ & $24.85(4.37)$ \\
$41-50$ & & \\
Body mass index $\left(\mathrm{kg} / \mathrm{m}^{2}\right)$ & $10(50 \%)$ & \\
Normoweight & $3(15 \%)$ & \\
Overweight & $4(20 \%)$ & $104.80(4.25)$ \\
Obese I & $3(15 \%)$ & $121.70(23.10)$ \\
Obese II & & \\
Fasting blood glucose $(\mathrm{mg} / \mathrm{dL})$ & & \\
Blood glucose 2 hours OGTT (mg/dL) &
\end{tabular}

Kolmogorov-Smirnov test found that data of levels HOMA-IR and HOMA-B in prediabetic patient were in a normal distribution $(\mathrm{p}>0.05)$. On prediabetics patients, mean of HOMA-IR was 4.12 (3.17) and mean of HOMA-B was 188.31 (155.13). Normal levels for HOMA-IR is less than 2.0, and for HOMA-B is 107 0r more.

\section{Discussion}

Insulin resistance started dysglycemic state in prediabetic and typed 2 diabetes mellitus. Cobb et al. stated that insulin resistance is a predictor of risk of metabolic syndrome and cardiovascular disease[11]. Tang et al. stated that insulin resistance is an important factor in prediabetic pathophysiology. So, assessment of insulin resistance by using HOMA-IR is the key for primary prevention of diabetes dan nowadays it has been a part of screening guideline in high-risk group [12].

In this study, we found a mean of HOMA-IR level at 4.12 (3.17) in the prediabetic patient. This is by Aguirre et al. who concluded that the level of HOMA-IR in prediabetic group consists of IFT group 4.53 (3.82) and IGT group 4.24 (3.21) [13]. The increase of insulin resistance in this study is in accordance with studies conducted by Nguyen and Mohtarin et al. Mohtarin et al. concluded that HOMA-IR level is higher in prediabetic compared to normoglycemic group [14], while Nguyen et al. stated that HOMA-IR level is significantly higher in prediabetic compared to normoglycemic groups [15].

Wallace et al. said that beta cell dysfunction is the decrease in its function as seen from abnormal beta cell response to blood glucose concentration [16]. In our study, the mean HOMAB level was 188.31 (155.13) in the prediabetic patient. This was by Aguirre et al. who concluded that mean HOMA-B level in the prediabetic group was 169 (164). [13] The increase of HOMA- 
B in our study is following works by Mohtarin and Owei. Mohtarin et al. (2015) stated that HOMA-B level was significantly higher in 32 prediabetic patients (135) compared to 30 normoglycemic groups (125) [14]. Owei et al. (2017) found that mean HOMA-B level was significantly higher in prediabetic patients, 132 (57.1) compared to the normoglycemic group, 62.4 (62.7) [17].

Koh said that calculation of HOMA-B shows the increase of insulin secretion when the random blood glucose level is $180 \mathrm{mg} / \mathrm{dL}$ or more. HOMA-B level should be interpreted together with HOMA-IR. This is because increasing insulin sensitivity or decreasing insulin resistance will decrease HOMA-B, which does not reflect the failure of the beta cell function $[18]$.

\section{Conclusion}

Assessment of HOMA-IR and HOMA-B in 20 prediabetic patients showed that they underwent increasing HOMA-IR which was compensated for by increasing HOMA-B.

\section{References}

[1] El Din U, Mona M, Dina O.: Uric Acid in the Pathogenesis of Metabolic, Renal, and Cardiovascular Diseases: A review. Egypt: CrossMark. p: 1-12 (2016).

[2] DeFronzo R, Roy E, Muhammad A.: Pathophysiologic Approach to Therapy in Patients With Type 2 Diabetes. Texas: Diabetes Journals. p: 1-12 (2013).

[3] Matfin G, Richard E.: Advances in the Treatment of Prediabetes. USA: Ther Adv Endocrinol Metab. p: $1-10$ (2010).

[4] Diabetes Research Wellness Foundation. What is Diabetes (2016)

[5] Pour O, Samuel D.: Prediabetes as A Therapeutic Target. Clinical Chemistry 57:2. p: 215-20 (2011).

[6] Nasrul E, Sofitri.: Hiperurisemia pada Pradiabetes. Diakses dalam Jurnal Andalas ISSN: 23017406. (2012). 